TITLE:

\title{
Exactly solvable birth and death processes
}

$\operatorname{AUTHOR}(\mathrm{S})$ :

Sasaki, Ryu

CITATION:

Sasaki, Ryu. Exactly solvable birth and death processes. Journal of Mathematical Physics 2009, 50(10): 103509.

ISSUE DATE:

2009-10

URL:

http://hdl.handle.net/2433/87411

RIGHT:

c 2009 American Institute of Physics. 


\title{
Exactly solvable birth and death processes
}

\author{
Ryu Sasaki ${ }^{\text {a) }}$ \\ Yukawa Institute for Theoretical Physics, Kyoto University, Kyoto 606-8502, Japan
}

(Received 28 May 2009; accepted 7 August 2009; published online 1 October 2009)

\begin{abstract}
Many examples of exactly solvable birth and death processes, a typical stationary Markov chain, are presented together with the explicit expressions of the transition probabilities. They are derived by similarity transforming exactly solvable "matrix" quantum mechanics, which is recently proposed by Odake and the author [S. Odake and R. Sasaki, J. Math. Phys. 49, 053503 (2008)]. The (q-) Askey scheme of hypergeometric orthogonal polynomials of a discrete variable and their dual polynomials play a central role. The most generic solvable birth/death rates are rational functions of $q^{x}$ (with $x$ being the population) corresponding to the $q$-Racah polynomial. () 2009 American Institute of Physics. [doi:10.1063/1.3215983]
\end{abstract}

\section{INTRODUCTION}

The Brownian motion, a typical stationary Markov process with a continuous state space, is known to be described well by the Fokker-Planck equation. ${ }^{1,2}$ A birth and death process, on the other hand, being a typical stationary Markov chain with a set of non-negative integers as a state space, ${ }^{2,3}$ can be naturally considered as a discretization of a one-dimensional (1D) Fokker-Planck equation. Although birth and death processes have a wide range of applications, ${ }^{2,3}$ such as demography, queueing theory, inventory models, and chemical dynamics, we will focus on their mathematical aspect, i.e., the exact solvability. In this paper, we present 18 exactly solvable birth and death processes based on the $(q-)$ Askey scheme of hypergeometric orthogonal polynomials having discrete orthogonality measures. They are also called orthogonal polynomials of a discrete variable. ${ }^{4-6}$ For example, they are the $(q-)$ Racah, the $(q-)$ (dual)Hahn, the $(q-)$ Krawtchouk, the $(q-)$ Charlier, and the $(q-)$ Meixner polynomials. ${ }^{4,5}$ Various expressions of the transition probability are given explicitly together with the totality of the eigenvalues and the measures of the Karlin-McGregor type representation. ${ }^{8}$

It is well known that the 1D Fokker-Planck equation is related by a similarity transformation to a corresponding 1D time-independent Schrödinger equation ${ }^{1}$ or the eigenvalue problem for a suitable Hamiltonian. In other words, solutions of an exactly solvable Schrödinger equation give the solutions of the corresponding Fokker-Planck equation, which is now exactly solvable. Exact solvability means that the totality of the eigenvalues (in these cases, all are discrete) and the corresponding eigenfunctions are obtained exactly. Here the Hamiltonian in quantum mechanics is a Hermitian (self-adjoint) linear operator in a certain Hilbert space. A natural discretization of the Hamiltonians of 1D quantum mechanics is Hermitian matrices of a finite or infinite dimensions. Recently, exactly solvable "matrix" quantum mechanics was proposed by Odake and the present author ${ }^{9}$ by adopting special types of tridiagonal Jacobi matrices of finite or infinite dimensions as Hamiltonians. The eigenfunctions are spanned by the abovementioned orthogonal polynomials of a discrete variable. The corresponding discretization of the Fokker-Planck equation is, as expected, the birth and death process with a reflecting wall(s) (3.20). Among the 18 exactly solvable birth and death processes to be explored in this paper, some are quite well known having the linear ${ }^{2,3,10,11}$ and quadratic ${ }^{12}$ birth and death rates, corresponding to the Meixner (Sec. IV B 1), Charlier (Sec. IV B 2), Krawtchouk (Sec. IV A 4), and Hahn (Sec. IV A 2) polynomials. The

\footnotetext{
${ }^{a)}$ Electronic mail: ryu@yukawa.kyoto-u.ac.jp.
} 
others have rational functions (of the population $x$ ) of the birth and death rates corresponding to the dual Hahn (Sec. IV A 3) and Racah (Sec. IV A 1) polynomials and some others have $q^{ \pm x}$-linear, quadratic, and rational birth and death rates. The most generic one is the $q$-Racah polynomial (Sec. IV A 5) having $q^{x}$ rational birth and death rates (4.21).

This paper is organized as follows. In Sec. II, the general properties of the Hamiltonians in 1D quantum mechanics (and/or the Hermitian matrices) are reviewed in Sec. II A. The relationship between the Schrödinger equation and the corresponding Fokker-Planck equation is recapitulated in Sec. II B and the solutions of the initial value problem of the Fokker-Planck equations and the transition probabilities are expressed in terms of the orthogonal polynomials constituting the eigenfunctions of the corresponding Schrödinger equation. In Sec. III the birth and death operator is derived from the generic form of the Hamiltonian of the exactly solvable matrix quantum mechanics of Ref. 9. The solutions of the initial value problem of the birth and death equations and the transition probabilities are expressed in terms of the orthogonal polynomials constituting the eigenfunctions of the corresponding Schrödinger equation of the matrix quantum mechanics. Various equivalent expressions of the transition probabilities are derived in terms of the dual polynomials. Section IV provides various data, the birth and death rates, the energy spectra and the sinusoidal coordinates, the stationary probability, the normalization constants, and the eigenpolynomials of the exactly solvable 18 models, which are sufficient to evaluate the transition probability explicitly. These 18 models are named after the eigenpolynomials, such as the $(q-)$ Racah, etc. Section V is for a brief summary and comments. The Appendix provides the collection of the definitions of basic symbols and functions for self-containedness. Throughout this paper we use the parameter $q$ in the range of $0<q<1$.

\section{FOKKER-PLANCK OPERATOR FROM HAMILTONIAN}

Here we recapitulate the well-known connection between the Fokker-Planck equation and the Schrödinger equation ${ }^{1}$ in order to introduce appropriate notation and settings for the main purpose of the paper; connecting the birth and death process to the matrix quantum mechanics to be explored in Sec. III.

\section{A. Properties of Hamiltonians}

Throughout this paper we discuss one degree of freedom systems only. The Hamiltonians to be discussed in this paper are time independent and share the properties listed below. Most properties are common to the Hamiltonians having the continuous dynamical variable $x$ (to be used for the Fokker-Planck equation) and the discrete dynamical variable $x$ (to be applied to the birth and death processes). They are expressed by the same symbols. When they need different symbols, such as the $L^{2}$ and $\ell^{2}$ norms, two different expressions are shown in a curly bracket as in (2.3) and (2.7). The upper (lower) one is for the continuous (discrete) dynamical variable case. The former (the continuous variable) case corresponds to the ordinary quantum mechanics and the "discrete" quantum mechanics with the pure imaginary shifts, ${ }^{13}$ which gives rise to the "deformed" Fokker-Planck equations. ${ }^{14}$

(i) Factorizability,

$$
\mathcal{H}=\mathcal{A}^{\dagger} \mathcal{A}
$$

in which ${ }^{\dagger}$ denotes the Hermitian conjugation with respect to the standard $L^{2}\left(\ell^{2}\right)$ inner product [see (2.3)]. This also means that the Hamiltonian $\mathcal{H}$ is positive semidefinite.

(ii) Completeness of its eigenfunctions $\phi_{n}(x)$ belonging to discrete eigenvalues (all distinct),

$$
\mathcal{H} \phi_{n}(x)=\mathcal{E}(n) \phi_{n}(x), \quad \mathcal{E}(0)<\mathcal{E}(1)<\cdots,
$$

and all the eigenvectors are square normalizable and orthogonal with each other 


$$
\left(\phi_{n}, \phi_{m}\right)=\left\{\begin{array}{c}
\operatorname{def} \phi_{n}(x)^{*} \phi_{m}(x) d x \\
\sum_{x} \phi_{n}(x)^{*} \phi_{m}(x)
\end{array}\right\}=\frac{1}{d_{n}^{2}} \delta_{n m}, \quad 0<d_{n}<\infty .
$$

The range of the integration (summation) depends on the specific Hamiltonian. Any element in the Hilbert space $\mathbf{H}$ is expanded by $\left\{\phi_{n}\right\}$,

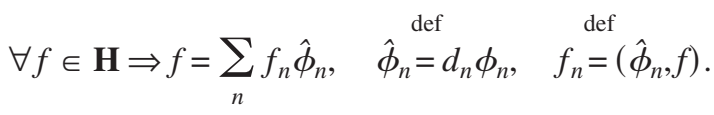

Here and hereafter, $\hat{f}$ denotes a normalized vector $\hat{f}=f / \sqrt{(f, f)}$. We choose all the eigenfunctions $\left\{\phi_{n}\right\}$ to be real, which is always possible in 1D quantum mechanics.

(iii) The ground state wave function $\phi_{0}$ is annihilated by $\mathcal{A}$ and is positive everywhere,

$$
\mathcal{A} \phi_{0}(x)=0 \Rightarrow \mathcal{H} \phi_{0}(x)=0, \quad \mathcal{E}(0)=0, \quad \phi_{0}(x)>0 .
$$

(iv) The eigenfunction $\phi_{n}(x)$ is $\phi_{0}(x)$ times a polynomial,

$$
\phi_{n}(x)=\phi_{0}(x) P_{n}(\eta(x)), \quad n=0,1,2, \ldots, \quad P_{0} \equiv 1,
$$

in which a real function $\eta(x)$ is called a sinusoidal coordinate. ${ }^{15,9,13}$ In other words, $P_{n}(\eta)$ is an orthogonal polynomial with the orthogonality measure $\phi_{0}(x)^{2}$,

$$
\left\{\begin{array}{c}
\int \phi_{0}(x)^{2} P_{n}(\eta(x)) P_{m}(\eta(x)) d x \\
\sum_{x} \phi_{0}(x)^{2} P_{n}(\eta(x)) P_{m}(\eta(x))
\end{array}\right\}=\frac{1}{d_{n}^{2}} \delta_{n m} .
$$

(v) The similarity transformed Hamiltonian $\mathcal{H}$ with respect to $\phi_{0}(x)$

$$
\widetilde{\mathcal{H}}=\phi_{0}^{-1} \circ \mathcal{H} \circ \phi_{0}
$$

provides a differential (difference) equation governing the polynomial $P_{n}(\eta(x))$.

\section{B. Fokker-Planck equation}

The Fokker-Planck equation in one dimension reads as

$$
\frac{\partial}{\partial t} \mathcal{P}(x ; t)=L_{\mathrm{FP}} \mathcal{P}(x ; t), \quad \mathcal{P}(x ; t) \geq 0, \quad \int \mathcal{P}(x ; t) d x=1,
$$

in which $\mathcal{P}(x ; t)$ is the probability distribution over certain continuous range of the parameter $x$; for example, $(-\infty, \infty),(0, \infty)$, or $(0, \pi)$. The Fokker-Planck operator $L_{\mathrm{FP}}$ corresponding to the Hamiltonian $\mathcal{H}(2.2)$ is defined by ${ }^{1,14}$

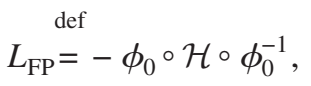

in which $\phi_{0}$ is defined in (2.5). (It should be emphasized that the inverse similarity transformation in terms of $\phi_{0}$ is used here: $L_{\mathrm{FP}}=-\phi_{0}^{2} \circ \tilde{\mathcal{H}} \circ \phi_{0}^{-2}$.) This guarantees that the eigenvalues of $L_{\mathrm{FP}}$ are negative semidefinite. The square normalized ground state eigenfunction $\phi_{0}(x)$ provides the stationary distribution $\hat{\phi}_{0}(x)^{2}$ of the corresponding Fokker-Planck operator, 


$$
\frac{\partial}{\partial t} \hat{\phi}_{0}(x)^{2}=L_{\mathrm{FP}} \hat{\phi}_{0}(x)^{2}=0, \quad \int \hat{\phi}_{0}(x)^{2} d x=1 .
$$

It is obvious that $\phi_{0}(x) \phi_{n}(x)$ is the eigenvector of the Fokker-Planck operator $L_{\mathrm{FP}}$,

$$
L_{\mathrm{FP}} \phi_{0}(x) \phi_{n}(x)=-\mathcal{E}(n) \phi_{0}(x) \phi_{n}(x), \quad n=0,1, \ldots
$$

Corresponding to an arbitrary initial probability distribution $\mathcal{P}(x ; 0)$ [with $\int \mathcal{P}(x ; 0) d x=1$ ], which can be expressed as a linear combination of $\left\{\hat{\phi}_{0}(x) \hat{\phi}_{n}(x)\right\}, n=0,1, \ldots$,

$$
\mathcal{P}(x ; 0)=\hat{\phi}_{0}(x) \sum_{n=0}^{\infty} c_{n} \hat{\phi}_{n}(x), \quad c_{0}=1, \quad c_{n}=\left(\hat{\phi}_{n}, \hat{\phi}_{0}(x)^{-1} \mathcal{P}(x ; 0)\right), \quad n=1,2, \ldots,
$$

we obtain the solution of the Fokker-Planck equation,

$$
\mathcal{P}(x ; t)=\hat{\phi}_{0}(x) \sum_{n=0}^{\infty} c_{n} e^{-\mathcal{E}(n) t} \hat{\phi}_{n}(x), \quad t>0 .
$$

This is a consequence of the completeness of the eigenfunctions $\left\{\phi_{n}(x)\right\}$ (the polynomials) of the Hamiltonian $\mathcal{H}$. The positivity of the spectrum $\mathcal{E}(n)>0, n \geq 1(2.2)$ guarantees that the stationary distribution $\hat{\phi}_{0}^{2}(x)$ is achieved at future infinity,

$$
\lim _{t \rightarrow \infty} \mathcal{P}(x ; t)=\hat{\phi}_{0}^{2}(x) .
$$

The transition probability from $y$ at $t=0$ [i.e., $\mathcal{P}(x ; 0)=\delta(x-y)]$ to $x$ at $t$ is given by

$$
\mathcal{P}(y, x ; t)=\hat{\phi}_{0}(x) \hat{\phi}_{0}(y)^{-1} \sum_{n=0}^{\infty} e^{-\mathcal{E}(n) t} \hat{\phi}_{n}(x) \hat{\phi}_{n}(y), \quad t>0 .
$$

In terms of the polynomial $P_{n}(\eta(x))(2.6)$, it is expressed as

$$
\mathcal{P}(y, x ; t)=\phi_{0}(x)^{2} \sum_{n=0}^{\infty} d_{n}^{2} e^{-\mathcal{E}(n) t} P_{n}(\eta(x)) P_{n}(\eta(y)), \quad t>0,
$$

in which $d_{n}$ is the normalization constants (2.3) and (2.7).

As shown in Ref. 14 in some detail, various examples of exactly solvable quantum mechanics ${ }^{16,17}$ and the discrete quantum mechanics with the pure imaginary shifts ${ }^{13,18,15}$ provide many explicit cases in which the transition probability (2.16) and (2.17) can be obtained exactly. The corresponding orthogonal polynomials are the Hermite, Laguerre, and Jacobi polynomials in the ordinary quantum mechanics ${ }^{16,17}$ and the Meixner-Pollaczek, continuous (dual) Hahn, Wilson, and Askey-Wilson polynomials ${ }^{14,13}$ and their degenerate polynomials, such as the continuous $q$-Hermite polynomials.

\section{BIRTH AND DEATH PROCESS FROM MATRIX QUANTUM MECHANICS}

The birth and death equation is a discretization of the Fokker-Planck equation in one dimension (2.9). It reads as

$$
\frac{\partial}{\partial t} \mathcal{P}(x ; t)=\left(L_{\mathrm{BD}} \mathcal{P}\right)(x ; t), \quad \mathcal{P}(x ; t) \geq 0, \quad \sum_{x} \mathcal{P}(x ; t)=1,
$$

in which $\mathcal{P}(x ; t)$ is the probability distribution over a certain discrete set of the parameter $x$. Here, we simply take a set of consecutive non-negative integers, either finite or infinite,

$$
x \in \mathbb{Z}, \quad x \in[0, N] \text { or }[0, \infty) .
$$


The exactly solvable birth and death operator or a matrix $L_{\mathrm{BD}}$ is derived from the generic form of an exactly solvable Hamiltonian $\mathcal{H}$ of a discrete quantum mechanics with real shifts,

$$
\mathcal{H}=-\sqrt{B(x)} e^{\partial} \sqrt{D(x)}-\sqrt{D(x)} e^{-\partial} \sqrt{B(x)}+B(x)+D(x),
$$

in which the two functions $B(x)$ and $D(x)$ are real and positive but vanish at the boundary,

$$
B(x)>0, \quad D(x)>0, \quad D(0)=0, \quad B(N)=0 \text { for the finite case. }
$$

The explicit forms of the functions $B(x)$ and $D(x)$ are given in each subsection of Sec. IV, which are named after the orthogonal polynomials appearing as the main part of the eigenfunctions. In the Hamiltonian (3.3) $e^{ \pm \partial}$ is a formal shift operator acting on a function $f$ of $x$ as

$$
\left(e^{ \pm \partial} f\right)(x)=f(x \pm 1) .
$$

Thus the Schrödinger equation $\mathcal{H} \psi(x)=\mathcal{E} \psi(x)$ is a difference equation with real shifts,

$$
\begin{gathered}
(B(x)+D(x)) \psi(x)-\sqrt{B(x) D(x+1)} \psi(x+1)-\sqrt{B(x-1) D(x)} \psi(x-1)=\mathcal{E} \psi(x), \\
x=0,1, \ldots,(N), \ldots
\end{gathered}
$$

The boundary condition $D(0)=0$ is necessary for the term $\psi(-1)$ not to appear, and $B(N)=0$ is necessary for the term $\psi(N+1)$ not to appear in the finite dimensional matrix case.

Although the Hamiltonian $\mathcal{H}$ (3.3) is presented in a difference operator form, it is, in fact, a real symmetric tridiagonal (Jacobi) matrix,

$$
\begin{gathered}
\mathcal{H}=\left(\mathcal{H}_{x, y}\right), \quad \mathcal{H}_{x, y}=\mathcal{H}_{y, x}, \\
\mathcal{H}_{x, y}=-\sqrt{B(x) D(x+1)} \delta_{x+1, y}-\sqrt{B(x-1) D(x)} \delta_{x-1, y}+(B(x)+D(x)) \delta_{x, y} .
\end{gathered}
$$

As mentioned above, the Hamiltonian is factorizable $(2.1), \mathcal{H}=\mathcal{A}^{\dagger} \mathcal{A}$,

$$
\mathcal{A}^{\dagger}=\sqrt{B(x)}-\sqrt{D(x)} e^{-\partial}, \quad \mathcal{A}=\sqrt{B(x)}-e^{\partial} \sqrt{D(x)} .
$$

In the matrix form, $\mathcal{A}^{\dagger}$ has the diagonal and subdiagonal elements only and $\mathcal{A}$ has the diagonal and superdiagonal elements only

$$
\left(\mathcal{A}^{\dagger}\right)_{x, y}=\sqrt{B(x)} \delta_{x, y}-\sqrt{D(x)} \delta_{x-1, y}, \quad \mathcal{A}_{x, y}=\sqrt{B(x)} \delta_{x, y}-\sqrt{D(x+1)} \delta_{x+1, y} .
$$

Equation (2.5) determining the ground state wave function $\phi_{0}$ is easy to solve since $\mathcal{A} \phi_{0}=0$ is a two term recurrence relation,

$$
\frac{\phi_{0}(x+1)}{\phi_{0}(x)}=\sqrt{\frac{B(x)}{D(x+1)}} .
$$

It can be solved elementarily with the boundary (initial) condition $\phi_{0}(0)=1$,

$$
\phi_{0}(x)=\sqrt{\prod_{y=0}^{x-1} \frac{B(y)}{D(y+1)}}, \quad x=1,2, \ldots
$$

With the standard convention $\prod_{k=n}^{n-1} *=1$, the expression (3.11) is valid for $x=0$, too. For the infinite matrix case, the requirement of the finite $\ell^{2}$ norm of the eigenvectors 


$$
\sum_{x=0}^{\infty} \phi_{0}(x)^{2}=\sum_{x=0}^{\infty} \prod_{y=0}^{x-1} \frac{B(y)}{D(y+1)}<\infty
$$

imposes constraints on the asymptotic behaviors of $B(x)$ and $D(x)$.

With the above explicit form of the ground state wave function $\phi_{0}(x)$, the similarity transformed Hamiltonian (2.8) is easily obtained

$$
\widetilde{\mathcal{H}}=\phi_{0}^{-1} \circ \mathcal{H} \circ \phi_{0}=B(x)\left(1-e^{\partial}\right)+D(x)\left(1-e^{-\partial}\right) .
$$

As mentioned above, $\tilde{\mathcal{H}}$ provides the difference equation for the polynomial eigenfunctions,

$$
\left(\tilde{\mathcal{H}} P_{n}\right)(\eta(x))=\mathcal{E}(n) P_{n}(\eta(x)),
$$

that is,

$$
B(x)\left(P_{n}(\eta(x))-P_{n}(\eta(x+1))\right)+D(x)\left(P_{n}(\eta(x))-P_{n}(\eta(x-1))\right)=\mathcal{E}(n) P_{n}(\eta(x)) .
$$

The eigenpolynomials $\left\{P_{n}\right\}$ are the orthogonal polynomials of a discrete variable. See Sec. 5 of Ref. 9 for various forms of $B(x)$ and $D(x)$ and the corresponding orthogonal polynomials. It is also recapitulated in Sec. IV of this paper. For example, they are the $\left(q^{-}\right)$Racah, the $\left(q^{-}\right)$(dual)Hahn, the $(q-)$ Krawtchouk, the $(q-)$ Charlier, and the $(q-)$ Meixner polynomials. ${ }^{4,5,7}$ As a matrix, $\widetilde{\mathcal{H}}$ is another tridiagonal matrix

$$
\widetilde{\mathcal{H}}=\left(\tilde{\mathcal{H}}_{x, y}\right), \quad \widetilde{\mathcal{H}}_{x, y}=B(x)\left(\delta_{x, y}-\delta_{x+1, y}\right)+D(x)\left(\delta_{x, y}-\delta_{x-1, y}\right) .
$$

Corresponding to (2.10), the inverse similarity transformation of the Hamiltonian $\mathcal{H}$ supplies the birth and death operator $L_{\mathrm{BD}}$,

$$
L_{\mathrm{BD}} \stackrel{\text { def }}{=}-\phi_{0} \circ \mathcal{H} \circ \phi_{0}^{-1}=\left(e^{-\partial}-1\right) B(x)+\left(e^{\partial}-1\right) D(x) .
$$

Obviously, the stationary probability is given by $\hat{\phi}_{0}(x)^{2}=d_{0}^{2} \phi_{0}(x)^{2}$. In the matrix form, $L_{\mathrm{BD}}$ is again tridiagonal

$$
L_{\mathrm{BD}}=\left(L_{\mathrm{BD}_{x, y}}\right), \quad L_{\mathrm{BD}_{x, y}}=B(x-1) \delta_{x-1, y}-B(x) \delta_{x, y}+D(x+1) \delta_{x+1, y}-D(x) \delta_{x, y} .
$$

In fact, $-L_{\mathrm{BD}}$ is the transposed matrix of $\tilde{\mathcal{H}}$,

$$
-L_{\mathrm{BD}}=(\tilde{\mathcal{H}})^{t}, \quad-L_{\mathrm{BD}_{x, y}}=\tilde{\mathcal{H}}_{y, x} .
$$

With the explicit form of the birth and death operator $L_{\mathrm{BD}}$, the birth and death equation (3.1) in our notation reads as

$$
\frac{\partial}{\partial t} \mathcal{P}(x ; t)=\sum_{y} L_{\mathrm{BD}_{x, y}} \mathcal{P}(y ; t)=-(B(x)+D(x)) \mathcal{P}(x ; t)+B(x-1) \mathcal{P}(x-1 ; t)+D(x+1) \mathcal{P}(x+1 ; t) .
$$

The standard interpretation is that $x$ is the population of a group, $\mathcal{P}(x ; t)$ is the probability for the group to have the population $x$ at the time $t, B(x)$ is the birth rate, and $D(x)$ is the death rate when the population is $x$. It is quite easy to remember. This is to be compared to the standard notation, for example ${ }^{8}$ (Sec. XVII.5 of Ref. 2 and Sec. 5.2 of Ref. 5),

$$
\frac{\partial}{\partial t} p_{n}(t)=-\left(\lambda_{n}+\mu_{n}\right) p_{n}(t)+\lambda_{n-1} p_{n-1}(t)+\mu_{n+1} p_{n+1}(t)
$$


TABLE I. Translation table.

\begin{tabular}{lcc}
\hline \hline & Standard $^{\mathrm{a}}$ & This paper \\
\hline Population & $n=0,1, \ldots,(N), \ldots$ & $x=0,1, \ldots,(N), \ldots$ \\
Probability & $p_{n}(t)$ & $\mathcal{P}(x ; t)$ \\
Birth rate & $\lambda_{n}\left(\lambda_{N}=0\right)$ & $B(x)(B(N)=0)$ \\
Death rate & $\mu_{n}\left(\mu_{0}=0\right)$ & $D(x)(D(0)=0)$ \\
\hline
\end{tabular}

${ }^{\mathrm{a}}$ References 2 and 5 .

in which $\lambda_{n}$ is the birth rate and $\mu_{n}$ is the death rate. The above translation table of the notation will be helpful (see Table I).

The boundary condition for the finite case, $\lambda_{N}=0(B(N)=0)(3.4)$ is said that the system has a reflecting wall at the population $N$.

The transition probability from $y$ at $t=0$ [i.e., $\mathcal{P}(x ; 0)=\delta_{x, y}$ ] to $x$ at $t$ has exactly the same expression as that in the Fokker-Planck equation (2.16),

$$
\mathcal{P}(y, x ; t)=\hat{\phi}_{0}(x) \hat{\phi}_{0}(y)^{-1} \sum_{n=0} e^{-\mathcal{E}(n) t} \hat{\phi}_{n}(x) \hat{\phi}_{n}(y), \quad t>0 .
$$

In terms of the polynomial $P_{n}(\eta(x))(2.6)$, it is expressed as

$$
\mathcal{P}(y, x ; t)=\phi_{0}(x)^{2} \sum_{n=0} d_{n}^{2} e^{-\mathcal{E}(n) t} P_{n}(\eta(x)) P_{n}(\eta(y)), \quad t>0 .
$$

It should be emphasized that in these formulas (3.22) and (3.23) everything is known including the measure in contradistinction to the general formula by Karlin-McGregor. ${ }^{8}$

Let us mention several equivalent expressions of the transition probability (3.23) in terms of the dual polynomials. ${ }^{19-21,9}$ It is well known that with proper normalization

$$
\eta(0)=0=\mathcal{E}(0), \quad P_{0} \equiv 1 \equiv Q_{0}, \quad P_{n}(0)=Q_{x}(0)=1,
$$

the two polynomials, $\left\{P_{n}(\eta)\right\}$, and its dual polynomial, $\left\{Q_{x}(\mathcal{E})\right\}$, coincide at the integer lattice points $^{9}$

$$
P_{n}(\eta(x))=Q_{x}(\mathcal{E}(n)), \quad n=0,1, \ldots,(N), \ldots, \quad x=0,1, \ldots,(N), \ldots .
$$

The dual polynomial $\left\{Q_{x}(\mathcal{E}(n))\right\}, x=0,1, \ldots$, is a right eigenvector of the similarity transformed Hamiltonian $\widetilde{\mathcal{H}}$ matrix with the eigenvalue $\mathcal{E}(n)$,

$$
\sum_{y} \tilde{\mathcal{H}}_{x, y} Q_{y}(\mathcal{E}(n))=\mathcal{E}(n) Q_{x}(\mathcal{E}(n)) .
$$

The above equation is the three term recurrence relation for the dual polynomials $\left\{Q_{x}(\mathcal{E})\right\}$,

$$
\begin{gathered}
(B(x)+D(x)) Q_{x}(\mathcal{E}(n))-B(x) Q_{x+1}(\mathcal{E}(n))-D(x) Q_{x-1}(\mathcal{E}(n))=\mathcal{E}(n) Q_{x}(\mathcal{E}(n)), \\
Q_{0}=1, \quad Q_{1}(\mathcal{E})=(B(0)-\mathcal{E}) / B(0), \quad Q_{2}(\mathcal{E})=(B(0)-\mathcal{E})(B(1)+D(1)-\mathcal{E}) /(B(0) B(1)), \ldots .
\end{gathered}
$$

For historical reasons, this polynomial $Q_{x}(\mathcal{E})$ is called the birth and death polynomial or the Karlin-McGregor polynomial. ${ }^{8}$

In terms of the dual polynomials or the Karlin-McGregor polynomial, the transition probability is 


$$
\mathcal{P}(y, x ; t)=\phi_{0}(x)^{2} \sum_{n=0} d_{n}^{2} e^{-\mathcal{E}(n) t} Q_{x}(\mathcal{E}(n)) Q_{y}(\mathcal{E}(n)), \quad t>0 .
$$

Following Ref. 5, let us introduce

$$
F_{x}(\mathcal{E}(n))=\phi_{0}(x)^{2} Q_{x}(\mathcal{E}(n)) .
$$

Since $L_{\mathrm{BD}}$ and $\tilde{\mathcal{H}}$ are related by

$$
L_{\mathrm{BD}}=-\phi_{0}^{2} \circ \tilde{\mathcal{H}} \circ \phi_{0}^{-2}
$$

it is easy to see that $F_{x}(\mathcal{E}(n))$ is a left eigenvector of $\tilde{\mathcal{H}}$ and thus a right eigenvector of the birth and death operator $L_{\mathrm{BD}}$,

$$
\sum_{y} L_{\mathrm{BD}_{x, y}} F_{y}(\mathcal{E}(n))=-\phi_{0}(x)^{2} \sum_{y} \tilde{\mathcal{H}}_{x, y} Q_{y}(\mathcal{E}(n))=-\mathcal{E}(n) \phi_{0}(x)^{2} Q_{x}(\mathcal{E}(n))=-\mathcal{E}(n) F_{x}(\mathcal{E}(n)) .
$$

In terms of the right eigenvectors of $L_{\mathrm{BD}}$, we obtain another expression of the transition probability $^{5}$

$$
\mathcal{P}(y, x ; t)=\frac{1}{\phi_{0}(y)^{2}} \sum_{n=0} d_{n}^{2} e^{-\mathcal{E}(n) t} F_{x}(\mathcal{E}(n)) F_{y}(\mathcal{E}(n)), \quad t>0 .
$$

The explicit forms of the transition probability (3.22), (3.23), (3.29), and (3.33) can be evaluated straightforwardly if the Hamiltonian $\mathcal{H}$ of an exactly solvable discrete quantum mechanics is given. Thus we may call the functions $B(x)$ and $D(x)$ in the Hamiltonian $\mathcal{H}$ of an exactly solvable discrete quantum mechanics (3.3) the birth and death rates of an exactly solvable birth and death process. As mentioned above, the association of the birth and death rates and the orthogonal polynomial in this paper and in literatures $8,5,12$ are dual to each other. Therefore the names of the polynomials in Sec. IV are the dual of the corresponding Karlin-McGregor polynomial except for the self-dual cases of the Krawtchouk (Sec. IV A 4), Meixner (Sec. IV B 1), and Charlier (Sec. IV B 2).

In Sec. IV we will present 18 examples of exactly solvable birth and death processes.

\section{18 EXAMPLES}

Now let us proceed to give the 18 explicit examples of exactly solvable birth and death processes. The input is simply the function forms of the birth and death rates $B(x)$ and $D(x)$. The rest is calculable. However, here we also provide other data, taken from Ref. 9, such as the energy eigenvalue $\mathcal{E}(n)$, the sinusoidal coordinate $\eta(x)$, the unnormalized stationary probability $\phi_{0}(x)^{2}$, the normalization constants $d_{n}^{2}$, and the polynomials $P_{n}(\eta)$. Following the order of our previous work on the exactly solvable discrete quantum mechanics, ${ }^{9}$ we handle the most generic one first and then followed by the simpler ones. There is a logical reason for this order. The simpler ones are usually obtained by specializing or restricting the parameters of the generic ones. Each example is called by the name of the corresponding orthogonal polynomial $P_{n}(\eta)$ with the number, e.g., [KS3.2] attached to it indicating the subsection in the standard review of Koekoek and Swarttouw. ${ }^{7}$ The finite $(N)$ cases are discussed first and then the infinite ones. In each group the Askey scheme of hypergeometric orthogonal polynomials (non- $q$ polynomials) will be discussed first and followed by the $q$-scheme polynomials.

Please note that the set of parameters is slightly different from the conventional ones ${ }^{4,5,7}$ for some polynomials, the reason explained in Ref. 9. For some polynomials, for example, the $(q-)$ Racah, (dual, $q^{-}$) Hahn, etc., there are many nonequivalent parametrizations of $B(x)$ and $D(x)$, which could lead to nonequivalent birth and death processes. Here we give only one of them as a representative since the purpose of the paper is to show exactly solvable structure not to provide 
an exhaustive list of all solvable models. See Ref. 9 for more general parametrizations and the allowed ranges of the parameters. In the same spirit, we did not include some of the polynomials listed in Ref. 9.

\section{A. Finite dimensional cases}

\section{Racah [KS1.2]}

The Racah polynomial is the most generic hypergeometric orthogonal polynomial of a discrete variable. All the other (non- $q$ ) polynomials are obtained by restriction or limiting procedure. The function $B(x)$ and $D(x)$ depend on four real parameters $a, b, c$, and $d$, with one of them, say $c$, being related to $N, c \equiv-N$,

$$
B(x)=-\frac{(x+a)(x+b)(x+c)(x+d)}{(2 x+d)(2 x+1+d)}, \quad D(x)=-\frac{(x+d-a)(x+d-b)(x+d-c) x}{(2 x-1+d)(2 x+d)} .
$$

The other data are

$$
\begin{gathered}
\mathcal{E}(n)=n(n+\tilde{d}), \quad \eta(x)=x(x+d), \quad \tilde{d}=a+b+c-d-1, \\
a \geq b, \quad d>0, \quad a>N+d, \quad 0<b<1+d, \\
\phi_{0}(x)^{2}=\frac{(a, b, c, d)_{x}}{(1+d-a, 1+d-b, 1+d-c, 1)_{x}} \frac{2 x+d}{d}, \\
d_{n}^{2}=\frac{(a, b, c, \tilde{d})_{n}}{(1+\tilde{d}-a, 1+\tilde{d}-b, 1+\tilde{d}-c, 1)_{n}} \frac{2 n+\tilde{d}}{\tilde{d}} \times \frac{(-1)^{N}(1+d-a, 1+d-b, 1+d-c)_{N}}{(\tilde{d}+1)_{N}(d+1)_{2 N}} .
\end{gathered}
$$

Here $(a)_{n}$ is the Pochhammer symbol (A1). Throughout this section, the format for $d_{n}^{2}$ consists of two parts separated by a $\times$ symbol: $d_{n}^{2}=\left(d_{n}^{2} / d_{0}^{2}\right) \times d_{0}^{2}$. The second part $d_{0}^{2}$ satisfies the relation $\sum_{x} \phi_{0}(x)^{2}=1 / d_{0}^{2}$. The polynomial is

$$
P_{n}(\eta(x))={ }_{4} F_{3}\left(\begin{array}{c|c}
-n, n+\tilde{d},-x, x+d & 1 \\
a, b, c & \mid
\end{array}\right),
$$

in which ${ }_{4} F_{3}$ is the standard hypergeometric series (A3). The dual polynomial is again the Racah polynomial with the parameter correspondence $(a, b, c, d) \leftrightarrow(a, b, c, \widetilde{d})$. The rational (a quartic polynomial divided by a quadratic polynomial) birth and death rates (4.1) have not yet been discussed but the Racah polynomial appears in Ref. 12.

\section{Hahn [KS1.5]}

This is a well-known example of quadratic (in $x$ ) birth and death rates with two real positive parameters $a$ and $b$,

$$
B(x)=(x+a)(N-x), \quad D(x)=x(b+N-x) .
$$

It has a quadratic energy spectrum

$$
\mathcal{E}(n)=n(n+a+b-1), \quad \eta(x)=x, \quad \phi_{0}(x)^{2}=\frac{N !}{x !(N-x) !} \frac{(a)_{x}(b)_{N-x}}{(b)_{N}},
$$




$$
\begin{gathered}
d_{n}^{2}=\frac{N !}{n !(N-n) !} \frac{(a)_{n}(2 n+a+b-1)(a+b)_{N}}{(b)_{n}(n+a+b-1)_{N+1}} \times \frac{(b)_{N}}{(a+b)_{N}}, \\
P_{n}(\eta(x))={ }_{3} F_{2}\left(\begin{array}{c|c}
-n, n+a+b-1,-x & 1 \\
a,-N & 1
\end{array}\right) .
\end{gathered}
$$

The dual polynomial is the dual Hahn polynomial of Sec. IV A 3 The quadratic birth and death rates are discussed in Ref. 12 associated with the dual Hahn polynomial.

\section{Dual Hahn [KS1.6]}

The set of parameters is the same as the Hahn polynomial case. The birth and death rates are rational functions of $x$,

$$
B(x)=\frac{(x+a)(x+a+b-1)(N-x)}{(2 x-1+a+b)(2 x+a+b)}, \quad D(x)=\frac{x(x+b-1)(x+a+b+N-1)}{(2 x-2+a+b)(2 x-1+a+b)},
$$

giving rise to a linear energy spectrum

$$
\begin{gathered}
\mathcal{E}(n)=n, \quad \eta(x)=x(x+a+b-1), \quad \phi_{0}(x)^{2}=\frac{N !}{x !(N-x) !} \frac{(a)_{x}(2 x+a+b-1)(a+b)_{N}}{(b)_{x}(x+a+b-1)_{N+1}} \\
d_{n}^{2}=\frac{N !}{n !(N-n) !} \frac{(a)_{n}(b)_{N-n}}{(b)_{N}} \times \frac{(b)_{N}}{(a+b)_{N}} \\
P_{n}(\eta(x))={ }_{3} F_{2}\left(\begin{array}{c}
-n, x+a+b-1,-x \mid \\
a,-N
\end{array}\right)
\end{gathered}
$$

\section{Krawtchouk [KS1.10] (self-dual)}

The case of linear birth and death rates are a very well-known example (the Ehrenfest model $)^{11}$ of an exactly solvable birth and death processes ${ }^{2,3}$

$$
\begin{gathered}
B(x)=p(N-x), \quad D(x)=(1-p) x, \quad 0<p<1, \\
\mathcal{E}(n)=n, \quad \eta(x)=x, \\
\phi_{0}(x)^{2}=\frac{N !}{x !(N-x) !}\left(\frac{p}{1-p}\right)^{x}, \quad d_{n}^{2}=\frac{N !}{n !(N-n) !}\left(\frac{p}{1-p}\right) \times{ }^{n}(1-p)^{N}, \\
P_{n}(\eta(x))={ }_{2} F_{1}\left(\begin{array}{c}
-n,-x \mid p^{-1} \\
-N
\end{array}\right) .
\end{gathered}
$$

This is a simplest example of self-dual polynomials. The stationary probability $\phi_{0}(x)^{2} d_{0}^{2}$ is the binomial distribution.

\section{5. q-Racah [KS3.2]}

This is the first example of the $q$-scheme of the orthogonal polynomials. Among them the $q$-Racah polynomial is the most generic. The set of parameters is four real numbers $(a, b, c, d)$, which is different from the standard one in the same manner as for the Racah polynomial. We restrict them 


$$
c=q^{-N}, \quad a \leq b, \quad 0<d<1, \quad 0<a<q^{N} d, \quad q d<b<1, \quad \tilde{d}<q^{-1}, \quad \tilde{d}=a b c d^{-1} q^{-1} .
$$

The functions $B(x)$ and $D(x)$ are

$$
\begin{gathered}
B(x)=-\frac{\left(1-a q^{x}\right)\left(1-b q^{x}\right)\left(1-c q^{x}\right)\left(1-d q^{x}\right)}{\left(1-d q^{2 x}\right)\left(1-d q^{2 x+1}\right)}, \\
D(x)=-\tilde{d} \frac{\left(1-a^{-1} d q^{x}\right)\left(1-b^{-1} d q^{x}\right)\left(1-c^{-1} d q^{x}\right)\left(1-q^{x}\right)}{\left(1-d q^{2 x-1}\right)\left(1-d q^{2 x}\right)} .
\end{gathered}
$$

The other data are

$$
\begin{gathered}
\mathcal{E}(n)=\left(q^{-n}-1\right)\left(1-\tilde{d} q^{n}\right), \quad \eta(x)=\left(q^{-x}-1\right)\left(1-d q^{x}\right), \\
\phi_{0}(x)^{2}=\frac{(a, b, c, d ; q)_{x}}{\left(a^{-1} d q, b^{-1} d q, c^{-1} d q, q ; q\right)_{x} \tilde{d}^{x}} \frac{1-d q^{2 x}}{1-d}, \\
d_{n}^{2}=\frac{(a, b, c, \tilde{d} ; q)_{n}}{\left(a^{-1} \tilde{d} q, b^{-1} \tilde{d} q, c^{-1} \tilde{d} q, q ; q\right)_{n} d^{n}} \frac{1-\tilde{d} q^{2 n}}{1-\tilde{d}} \times \frac{(-1)^{N}\left(a^{-1} d q, b^{-1} d q, c^{-1} d q ; q\right)_{N} \tilde{d}^{N} q^{1 / 2 N(N+1)}}{(\tilde{d} q ; q)_{N}(d q ; q)_{2 N}}, \\
P_{n}(\eta(x))={ }_{4} \phi_{3}\left(\begin{array}{c}
q^{-n}, \tilde{d} q^{n}, q^{-x}, d q^{x} \\
a, b, c
\end{array} \mid \begin{array}{c}
q ; q),
\end{array}\right.
\end{gathered}
$$

in which ${ }_{4} \phi_{3}$ is the basic hypergeometric series (A4) and $(a ; q)_{n}$ is the $q$-Pochhammer symbol (A2). The dual $q$-Racah polynomial is again the $q$-Racah polynomial with the parameter correspondence $(a, b, c, d) \leftrightarrow(a, b, c, \widetilde{d})$.

\section{6. q-Hahn [KS3.6]}

The $q$-Hahn polynomial has two positive parameters $a$ and $b$ and the birth and death rates are quadratic polynomials in $q^{x}$,

$$
B(x)=\left(1-a q^{x}\right)\left(q^{x-N}-1\right), \quad D(x)=a q^{-1}\left(1-q^{x}\right)\left(q^{x-N}-b\right), \quad 0<a, \quad b<1 .
$$

The other data are

$$
\begin{gathered}
\mathcal{E}(n)=\left(q^{-n}-1\right)\left(1-a b q^{n-1}\right), \quad \eta(x)=q^{-x}-1, \\
\phi_{0}(x)^{2}=\frac{(q ; q)_{N}}{(q ; q)_{x}(q ; q)_{N-x}} \frac{(a ; q)_{x}(b ; q)_{N-x}}{(b ; q)_{N} a^{x}}, \\
d_{n}^{2}=\frac{(q ; q)_{N}}{(q ; q)_{n}(q ; q)_{N-n}} \frac{\left(a, a b q^{-1} ; q\right)_{n}}{\left(a b q^{N}, b ; q\right)_{n} a^{n}} \frac{1-a b q^{2 n-1}}{1-a b q^{-1}} \times \frac{(b ; q)_{N} a^{N}}{(a b ; q)_{N}}, \\
P_{n}(\eta(x))={ }_{3} \phi_{2}\left(\begin{array}{c}
q^{-n}, a b q^{n-1}, q^{-x} \\
a, q^{-N}
\end{array} \mid q ; q\right) .
\end{gathered}
$$

Obviously the $q$-Hahn and dual $q$-Hahn are dual to each other. 


\section{Dual q-Hahn [KS3.7]}

For obvious reasons, we adopt the same parameters $(a, b)$ for the $q$-Hahn and dual $q$-Hahn polynomials. The birth and death rates are rational functions of $q^{x}$,

$$
\begin{gathered}
B(x)=\frac{\left(q^{x-N}-1\right)\left(1-a q^{x}\right)\left(1-a b q^{x-1}\right)}{\left(1-a b q^{2 x-1}\right)\left(1-a b q^{2 x}\right)}, \quad 0<a, \quad b<1, \\
D(x)=a q^{x-N-1} \frac{\left(1-q^{x}\right)\left(1-a b q^{x+N-1}\right)\left(1-b q^{x-1}\right)}{\left(1-a b q^{2 x-2}\right)\left(1-a b q^{2 x-1}\right)}, \\
\mathcal{E}(n)=q^{-n}-1, \quad \eta(x)=\left(q^{-x}-1\right)\left(1-a b q^{x-1}\right), \\
\phi_{0}(x)^{2}=\frac{(q ; q)_{N}}{(q ; q)_{x}(q ; q)_{N-x}} \frac{\left(a, a b q^{-1} ; q\right)_{x}}{\left(a b q^{N}, b ; q\right)_{x} a^{x}} \frac{1-a b q^{2 x-1}}{1-a b q^{-1}}, \\
d_{n}^{2}=\frac{(q ; q)_{N}}{(q ; q)_{n}(q ; q)_{N-n}} \frac{(a ; q)_{n}(b ; q)_{N-n}}{(b ; q)_{N} a^{n}} \times \frac{(b ; q)_{N} a^{N}}{(a b ; q)_{N}}, \\
P_{n}(\eta(x))={ }_{3} \phi_{2}\left(\begin{array}{c}
q^{-n}, a b q^{x-1}, q^{-x} \\
a, q^{-N}
\end{array} \mid q ; q\right) .
\end{gathered}
$$

\section{Quantum q-Krawtchouk [KS3.14]}

This has one positive parameter $p>q^{-N}$. The birth and death rates are quadratic polynomials in $q^{x}$,

$$
\begin{gathered}
B(x)=p^{-1} q^{x}\left(q^{x-N}-1\right), \quad D(x)=\left(1-q^{x}\right)\left(1-p^{-1} q^{x-N-1}\right), \\
\mathcal{E}(n)=1-q^{n}, \quad \eta(x)=q^{-x}-1, \\
\phi_{0}(x)^{2}=\frac{(q ; q)_{N}}{(q ; q)_{x}(q ; q)_{N-x}} \frac{p^{-x} q^{x(x-1-N)}}{\left(p^{-1} q^{-N} ; q\right)_{x}}, \\
d_{n}^{2}=\frac{(q ; q)_{N}}{(q ; q)_{n}(q ; q)_{N-n}} \frac{p^{-n} q^{-N n}}{\left(p^{-1} q^{-n} ; q\right)_{n}} \times\left(p^{-1} q^{-N} ; q\right)_{N}, \\
P_{n}(\eta(x))={ }_{2} \phi_{1}\left(\begin{array}{c}
q^{-n}, q^{-x} \\
q^{-N}
\end{array} \mid q ; p q^{n+1}\right) .
\end{gathered}
$$

\section{9. q-Krawtchouk [KS3.15]}

This has one positive parameter $p>0$ and the birth and death rates are linear in $q^{x}$,

$$
\begin{gathered}
B(x)=q^{x-N}-1, \quad D(x)=p\left(1-q^{x}\right), \\
\mathcal{E}(n)=\left(q^{-n}-1\right)\left(1+p q^{n}\right), \quad \eta(x)=q^{-x}-1,
\end{gathered}
$$




$$
\begin{gathered}
\phi_{0}(x)^{2}=\frac{(q ; q)_{N}}{(q ; q)_{x}(q ; q)_{N-x}} p^{-x} q^{1 / 2 x(x-1)-x N}, \\
d_{n}^{2}=\frac{(q ; q)_{N}}{(q ; q)_{n}(q ; q)_{N-n}} \frac{(-p ; q)_{n}}{\left(-p q^{N+1} ; q\right)_{n} p^{n} q^{1 / 2 n(n+1)}} \frac{1+p q^{2 n}}{1+p} \times \frac{p^{N} q^{1 / 2 N(N+1)}}{(-p q ; q)_{N}}, \\
P_{n}(\eta(x))={ }_{3} \phi_{2}\left(\begin{array}{c}
q^{-n}, q^{-x},-p q^{n} \\
q^{-N}, 0
\end{array} \mid q ; q\right) .
\end{gathered}
$$

\section{Affine q-Krawtchouk [KS3.16] (self-dual)}

This has one positive parameter $p$ and the birth and death rates are quadratic polynomials in $q^{x}$,

$$
\begin{gathered}
B(x)=\left(q^{x-N}-1\right)\left(1-p q^{x+1}\right), \quad D(x)=p q^{x-N}\left(1-q^{x}\right), \quad 0<p<q^{-1}, \\
\mathcal{E}(n)=q^{-n}-1, \quad \eta(x)=q^{-x}-1, \\
\phi_{0}(x)^{2}=\frac{(q ; q)_{N}}{(q ; q)_{x}(q ; q)_{N-x}} \frac{(p q ; q)_{x}}{(p q)^{x}}, \quad d_{n}^{2}=\frac{(q ; q)_{N}}{(q ; q)_{n}(q ; q)_{N-n}} \frac{(p q ; q)_{n}}{(p q)^{n}} \times(p q)^{N}, \\
P_{n}(\eta(x))={ }_{3} \phi_{2}\left(\begin{array}{c}
q^{-n}, q^{-x}, 0 \\
p q, q^{-N}
\end{array} \mid q ; q\right) .
\end{gathered}
$$

\section{B. Infinite dimensional cases}

In contrast to the finite dimensional case, the structure of the polynomials is severely constrained by the asymptotic forms of the functions $B(x)$ and $D(x)$ (3.12).

\section{Meixner [KS1.9] (self-dual)}

This is the best known example of exactly solvable birth and death processes ${ }^{10}$ and the birth and death rates are both linear in $x$ with simple linear energy spectra $\mathcal{E}(n)=n$ and $\eta(x)=x$. It has two positive parameters $\beta$ and $c$ :

$$
\begin{gathered}
B(x)=\frac{c}{1-c}(x+\beta), \quad D(x)=\frac{1}{1-c} x, \quad \beta>0, \quad 0<c<1, \\
\mathcal{E}(n)=n, \quad \eta(x)=x, \\
\phi_{0}(x)^{2}=\frac{(\beta)_{x} c^{x}}{x !}, \quad d_{n}^{2}=\frac{(\beta)_{n} c^{n}}{n !} \times(1-c)^{\beta}, \\
P_{n}(\eta(x))={ }_{2} F_{1}\left(\begin{array}{c}
-n,-x \\
\beta
\end{array} \mid 1-c^{-1}\right) .
\end{gathered}
$$

\section{Charlier [KS1.12] (self-dual)}

This is another best known example of exactly solvable birth and death processes with constant birth rates $a>0$ and linear death rates, 


$$
\begin{gathered}
B(x)=a, \quad D(x)=x, \\
\mathcal{E}(n)=n, \quad \eta(x)=x, \\
\phi_{0}(x)^{2}=\frac{a^{x}}{x !}, \quad d_{n}^{2}=\frac{a^{n}}{n !} \times e^{-a}, \\
P_{n}\left(\eta(x)={ }_{2} F_{0}\left(\begin{array}{c}
\left.-n,-x \mid-a^{-1}\right) . \\
-
\end{array}\right.\right.
\end{gathered}
$$

The stationary probability $\phi_{0}(x)^{2} d_{0}^{2}(4.57)$ is the Poisson distribution.

\section{3. little q-Jacobi [KS3.12]}

This has two parameters $a$ and $b$. The birth and death rates grow exponentially as $x$ tends to infinity,

$$
\begin{gathered}
B(x)=a\left(q^{-x}-b q\right), \quad D(x)=q^{-x}-1, \quad 0<a<q^{-1}, \quad b<q^{-1}, \\
\mathcal{E}(n)=\left(q^{-n}-1\right)\left(1-a b q^{n+1}\right), \quad \eta(x)=1-q^{x}, \\
\phi_{0}(x)^{2}=\frac{(b q ; q)_{x}}{(q ; q)_{x}}(a q)^{x}, \\
d_{n}^{2}=\frac{(b q, a b q ; q)_{n} a^{n} q^{n^{2}}}{(q, a q ; q)_{n}} \frac{1-a b q^{2 n+1}}{1-a b q} \times \frac{(a q ; q)_{\infty}}{\left(a b q^{2} ; q\right)_{\infty}}, \\
P_{n}(\eta(x))=(-a)^{-n} q^{-1 / 2 n(n+1)} \frac{(a q ; q)_{n}}{(b q ; q)_{n}}{ }_{2} \phi_{1}\left(\begin{array}{c}
q^{-n}, a b q^{n+1} \\
a q
\end{array} \mid q ; q^{x+1}\right) .
\end{gathered}
$$

The normalization of the polynomial is different from the conventional one.

\section{4. q-Meixner [KS3.13]}

This has two positive parameters $b$ and $c$. The birth and death rates are quadratic in $q^{x}$ and as $x$ goes to infinity, the birth rates tend to zero and the death rates tend to unity,

$$
\begin{gathered}
B(x)=c q^{x}\left(1-b q^{x+1}\right), \quad D(x)=\left(1-q^{x}\right)\left(1+b c q^{x}\right), \quad 0<b<q^{-1}, \quad c>0, \\
\mathcal{E}(n)=1-q^{n}, \quad \eta(x)=q^{-x}-1, \\
\phi_{0}(x)^{2}=\frac{(b q ; q)_{x}}{(q,-b c q ; q)_{x}} c^{x} q^{1 / 2 x(x-1)}, \quad d_{n}^{2}=\frac{(b q ; q)_{n}}{\left(q,-c^{-1} q ; q\right)_{n}} \times \frac{(-b c q ; q)_{\infty}}{(-c ; q)_{\infty}}, \\
P_{n}(\eta(x))={ }_{2} \phi_{1}\left(\begin{array}{c}
q^{-n}, q^{-x} \\
b q
\end{array} \mid q ;-c^{-1} q^{n+1}\right) .
\end{gathered}
$$

\section{Little q-Laguerre/Wall [KS3.20]}

This has one positive parameter $a$ and both the birth and death rates grow exponentially as $x$ tends to infinity, 


$$
\begin{gathered}
B(x)=a q^{-x}, \quad D(x)=q^{-x}-1, \quad 0<a<q^{-1}, \\
\mathcal{E}(n)=q^{-n}-1, \quad \eta(x)=1-q^{x}, \\
\phi_{0}(x)^{2}=\frac{(a q)^{x}}{(q ; q)_{x}}, \quad d_{n}^{2}=\frac{a^{n} q^{n^{2}}}{(q, a q ; q)_{n}} \times(a q ; q)_{\infty}, \\
P_{n}(\eta(x))={ }_{2} \phi_{0}\left(\begin{array}{c}
q^{-n}, q^{-x} \\
-
\end{array} \mid q ; a^{-1} q^{x}\right) .
\end{gathered}
$$

The normalization of the polynomial is different from the conventional one.

\section{Al-Salam-Carlitz II [KS3.25]}

This has one positive parameter $a$ and the birth and death rates are quadratic in $q^{x}$. As $x$ goes to infinity the birth rates tend to zero and death rates tend to unity,

$$
\begin{gathered}
B(x)=a q^{2 x+1}, \quad D(x)=\left(1-q^{x}\right)\left(1-a q^{x}\right), \quad 0<a<q^{-1}, \\
\mathcal{E}(n)=1-q^{n}, \quad \eta(x)=q^{-x}-1, \\
\phi_{0}(x)^{2}=\frac{a^{x} q^{x^{2}}}{(q, a q ; q)_{x}}, \quad d_{n}^{2}=\frac{(a q)^{n}}{(q ; q)_{n}} \times(a q ; q)_{\infty}, \\
P_{n}(\eta(x))={ }_{2} \phi_{0}\left(q^{-n}, q^{-x} \mid q ; a^{-1} q^{n}\right) .
\end{gathered}
$$

The normalization of the polynomial is different from the conventional one.

\section{Alternative q-Charlier [KS3.22]}

This has one positive parameter $a$. The birth rates are constant $a$, whereas the death rates grow exponentially as $x$ goes to infinity,

$$
\begin{gathered}
B(x)=a, \quad D(x)=q^{-x}-1, \quad a>0, \\
\mathcal{E}(n)=\left(q^{-n}-1\right)\left(1+a q^{n}\right), \quad \eta(x)=1-q^{x}, \\
\phi_{0}(x)^{2}=\frac{a^{x} q^{1 / 2 x(x+1)}}{(q ; q)_{x}}, d_{n}^{2}=\frac{a^{n} q^{1 / 2 n(3 n-1)}}{(q ; q)_{n}} \frac{(-a ; q)_{\infty}}{\left(-a q^{n} ; q\right)_{\infty}} \frac{1+a q^{2 n}}{1+a} \times \frac{1}{(-a q ; q)_{\infty}}, \\
P_{n}(\eta(x))=q^{n x}{ }_{2} \phi_{1}\left(\begin{array}{c}
q^{-n}, q^{-x} \\
0
\end{array} \mid q ;-a^{-1} q^{-n+1}\right) .
\end{gathered}
$$

The normalization of the polynomial is different from the conventional one.

\section{8. q-Charlier [KS3.23]}

This has one positive parameter $a$, and as $x$ goes to infinity, the birth rates tend to zero and the death rates tend to unity,

$$
B(x)=a q^{x}, \quad D(x)=1-q^{x}, \quad a>0,
$$




$$
\begin{gathered}
\mathcal{E}(n)=1-q^{n}, \quad \eta(x)=q^{-x}-1, \\
\phi_{0}(x)^{2}=\frac{a^{x} q^{1 / 2 x(x-1)}}{(q ; q)_{x}}, \quad d_{n}^{2}=\frac{q^{n}}{\left(-a^{-1} q, q ; q\right)_{n}} \times \frac{1}{(-a ; q)_{\infty}}, \\
P_{n}(\eta(x))={ }_{2} \phi_{1}\left(\begin{array}{c}
q^{-n}, q^{-x} \\
0
\end{array} \mid q ;-a^{-1} q^{n+1}\right) .
\end{gathered}
$$

\section{SUMMARY AND COMMENTS}

Following the simple line of arguments summarized in the following diagram, we presented 18 models of exactly solvable birth and death processes and their solutions, the transition probabilities. In the diagram "ES" stands for exactly solvable.

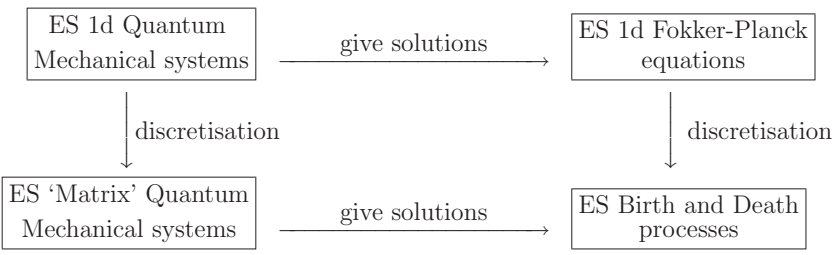

The exactly solvable matrix quantum mechanics or the $1 \mathrm{D}$ discrete quantum mechanics with real shifts was explored in detail in Ref. 9 to cover most of the hypergeometric orthogonal polynomials of a discrete variable in the $(q-)$ Askey scheme. ${ }^{4,5,7}$ For the "explanation" of the exact solvability, see a recent work. ${ }^{22}$ By comparing the present simple results with those in literatures $^{8,3,5,12}$ one would realize the essential role played by the energy spectrum $\mathcal{E}(n)$ and the sinusoidal coordinate $\eta(x)$. They are the eigenvalues of the two operators, called the Leonard pair, which characterize the orthogonal polynomials completely. ${ }^{19-21}$

In this paper we did not discuss the generalization of the birth and death processes which has $\mu_{0}>0(D(0)>0)$, the nonvanishing death rate at zero population, although this has led to a new type of orthogonal polynomials in the cases when the birth and death rates $B(x)$ and $D(x)$ are linear and quadratic in $x .^{23,24}$ It would be interesting to try further generalization in this direction for which $B(x)$ and $D(x)$ are rational, e.g., the Racah case (Sec. IV A 1) or $q$-linear, e.g., the $q$-Krawtchouk (Sec. IV A 9), or $q$-quadratic, e.g., the affine $q$-Krawtchouk (Sec. IV A 10), or even the $q$-rational, e.g., the $q$-Racah (Sec. IV A 5) cases.

It is a big challenge to try and find a closed form expression for

$$
\sum_{n=0} d_{n}^{2} e^{-\mathcal{E}(n) t} P_{n}(\eta(x)) P_{n}(\eta(y))
$$

appearing as a part of the transition probability (2.17) and (3.23) for various examples in section four. To the best of our knowledge, such expressions are known only for the linear energy spectrum $\mathcal{E}(n) \propto n$. For example, for the Fokker-Planck equation corresponding to the harmonic oscillator Hamiltonian, or the Ornshtein-Uhlenbeck process, ${ }^{1,14}$ we have

$$
\begin{gathered}
\mathcal{H}=-\frac{d^{2}}{d x^{2}}+x^{2}-1, \quad L_{\mathrm{FP}}=\frac{d^{2}}{d x^{2}}+2 \frac{d}{d x} x, \quad \mathcal{E}(n)=2 n, \quad \eta(x)=x, \\
\mathcal{P}(y, x ; t)=\frac{e^{-x^{2}}}{\sqrt{\pi}} \sum_{n=0}^{\infty} \frac{H_{n}(x) H_{n}(y)}{2^{n} n !} e^{-2 n t}=\frac{1}{\sqrt{\pi} \sqrt{1-e^{-4 t}}} \exp \left[-\frac{\left(x-y e^{-2 t}\right)^{2}}{1-e^{-4 t}}\right] .
\end{gathered}
$$

The last equality was derived based on (6.1.13) of Ref. 4. Another example is 


$$
\begin{gathered}
\mathcal{H}=-\frac{d^{2}}{d x^{2}}+x^{2}+\frac{g(g-1)}{x^{2}}-(1+2 g), \quad L_{\mathrm{FP}}=\frac{d^{2}}{d x^{2}}+2 \frac{d}{d x}\left(x-\frac{g}{x}\right), \\
\mathcal{E}_{n}=4 n, \quad \eta(x)=x^{2}, \quad \beta=g-1 / 2, \quad 0<x<\infty, \\
\mathcal{P}(y, x ; t)=2 e^{-x^{2}} x^{2 g} \sum_{n=0}^{\infty} \frac{n ! L_{n}^{(\beta)}\left(x^{2}\right) L_{n}^{(\beta)}\left(y^{2}\right)}{\Gamma(n+\beta+1)} e^{-4 n t} \\
=\frac{2 x^{2 g}}{\left(1-e^{-4 t}\right)} \exp \left[-\frac{\left(x^{2}+y^{2} e^{-4 t}\right)}{\left(1-e^{-4 t}\right)}\right]\left(x y e^{-2 t}\right)^{-\beta} I_{\beta}\left(\frac{2 x y e^{-2 t}}{1-e^{-4 t}}\right),
\end{gathered}
$$

in which $I_{\beta}$ is the modified Bessel function of the order of $\beta$. The last equality was derived based on (6.2.25) of Ref. 4. We would like to ask experts in special functions and orthogonal polynomials to derive such bilinear generating functions for various energy spectra,

$$
\mathcal{E}(n)=n(n+d), \quad q^{-n}-1, \quad 1-q^{n}, \quad\left(q^{-n}-1\right)\left(1-d q^{n}\right) .
$$

\section{ACKNOWLEDGMENTS}

We thank Mourad Ismail and Choon-Lin Ho who induced us to the present research. This work was supported in part by Grants-in-Aid for Scientific Research from the Ministry of Education, Culture, Sports, Science and Technology of Japan, Grant Nos. 18340061 and 19540179.

\section{APPENDIX: SOME DEFINITIONS RELATED TO THE HYPERGEOMETRIC AND $q$-HYPERGEOMETRIC FUNCTIONS}

For self-containedness we collect several definitions related to the $(q-)$ hypergeometric functions. ${ }^{7}$

- Pochhammer symbol $(a)_{n}$,

$$
(a)_{n}=\prod_{k=1}^{\operatorname{def}}(a+k-1)=a(a+1) \cdots(a+n-1)=\frac{\Gamma(a+n)}{\Gamma(a)} .
$$

- $q$-Pochhammer symbol $(a ; q)_{n}$,

$$
(a ; q)_{n}=\prod_{k=1}^{\operatorname{def} n}\left(1-a q^{k-1}\right)=(1-a)(1-a q) \cdots\left(1-a q^{n-1}\right) .
$$

- Hypergeometric series ${ }_{r} F_{s}$,

$$
{ }_{r} F_{s}\left(\begin{array}{c}
a_{1}, \ldots, a_{r} \\
b_{1}, \ldots, b_{s}
\end{array} \mid z\right)=\sum_{n=0}^{\operatorname{def}} \frac{\left(a_{1}, \ldots, a_{r}\right)_{n}}{\left(b_{1}, \ldots, b_{s}\right)_{n}} \frac{z^{n}}{n !},
$$

def

where $\left(a_{1}, \ldots, a_{r}\right)_{n}=\prod_{j=1}^{r}\left(a_{j}\right)_{n}=\left(a_{1}\right)_{n} \cdots\left(a_{r}\right)_{n}$.

- $q$-hypergeometric series (the basic hypergeometric series) ${ }_{r} \phi_{s}$, 


$$
{ }_{r} \phi_{s}\left(\begin{array}{l}
a_{1}, \cdots, a_{r} \\
b_{1}, \cdots, b_{s}
\end{array} \mid q ; z\right)=\sum_{n=0}^{\operatorname{def}} \frac{\left(a_{1}, \cdots, a_{r} ; q\right)_{n}}{\left(b_{1}, \cdots, b_{s} ; q\right)_{n}}(-1)^{(1+s-r) n} q^{(1+s-r) n(n-1) / 2} \frac{z^{n}}{(q ; q)_{n}},
$$

def

where $\left(a_{1}, \ldots, a_{r} ; q\right)_{n}=\prod_{j=1}^{r}\left(a_{j} ; q\right)_{n}=\left(a_{1} ; q\right)_{n} \cdots\left(a_{r} ; q\right)_{n}$.

${ }^{1}$ H. Risken, The Fokker-Planck Equation, 2nd ed. (Springer-Verlag, Berlin, 1996).

${ }^{2}$ W. Feller, An Introduction to Probability Theory and Its Applications, 2nd ed. (Wiley, New York, 1966), Vol. 1.

${ }^{3}$ W. Schoutens, Stochastic Processes and Orthogonal Polynomials, Lecture Notes in Statistics Vol. 146 (Springer, New York, 2000).

${ }^{4}$ G. E. Andrews, R. Askey, and R. Roy, Special Functions, Encyclopedia of Mathematics and Its Applications (The University Press, Cambridge, 1999).

${ }^{5}$ M. E. H. Ismail, Classical and Quantum Orthogonal Polynomials in One Variable, Encyclopedia of Mathematics and Its Applications (Cambridge University Press, Cambridge, 2005).

${ }^{6}$ A. F. Nikiforov, S. K. Suslov, and V. B. Uvarov, Classical Orthogonal Polynomials of a Discrete Variable (SpringerVerlag, Berlin, 1991).

${ }^{7}$ R. Koekoek and R. F. Swarttouw, e-print arXiv:math.CA/9602214.

${ }^{8}$ S. Karlin and J. L. McGregor, Trans. Am. Math. Soc. 85, 489 (1957).

${ }^{9}$ S. Odake and R. Sasaki, J. Math. Phys. 49, 053503 (2008).

${ }^{10}$ S. Karlin and J. L. McGregor, J. Math. Mech. 7, 643 (1958)

${ }^{11}$ S. Karlin and J. L. McGregor, J. Appl. Probab. 19, 477 (1965).

${ }_{12}^{12}$ W. van Assche, P. R. Parathasarathy, and R. B. Lenin, Math. Sci. 24, 105 (1999).

${ }^{13}$ S. Odake and R. Sasaki, Prog. Theor. Phys. 119, 663 (2008).

${ }^{14}$ C.-L. Ho and R. Sasaki, Prog. Theor. Phys. 118, 667 (2007); J. Math. Phys. 48, 073302 (2007).

${ }^{15}$ S. Odake and R. Sasaki, J. Math. Phys. 47, 102102 (2006); Phys. Lett. B 641, 112 (2006).

${ }^{16}$ L. Infeld and T. E. Hull, Rev. Mod. Phys. 23, 21 (1951).

${ }^{17}$ See, for example, a review F. Cooper, A. Khare, and U. Sukhatme, Phys. Rep. 251, 267 (1995).

${ }^{18}$ S. Odake and R. Sasaki, J. Nonlinear Math. Phys. 12, 507 (2005).

${ }^{19}$ D. Leonard, SIAM J. Math. Anal. 13, 656 (1982).

${ }^{20}$ E. Bannai and T. Ito, Algebraic Combinatorics I: Association Schemes (Benjamin/Cummings, Menlo Park, CA, 1984).

${ }^{21}$ P. Terwilliger, Numer. Linear Algebra Appl. 387, 235 (2004); Lecture notes for the summer school on orthogonal polynomials and special functions, Universidad Carlos III de Madrid, Leganes, Spain, July 8-18, 2004, e-print arXiv:math.QA/0408390

${ }^{22}$ S. Odake and R. Sasaki, e-print arXiv:0903.2604v1.

${ }^{23}$ R. Askey and J. Wimp, Proc. - R. Soc. Edinburgh, Sect. A: Math. 96, 15 (1984).

${ }^{24}$ M. E. H. Ismail, J. Lettessier, and G. Valent, SIAM J. Math. Anal. 20, 727 (1989). 